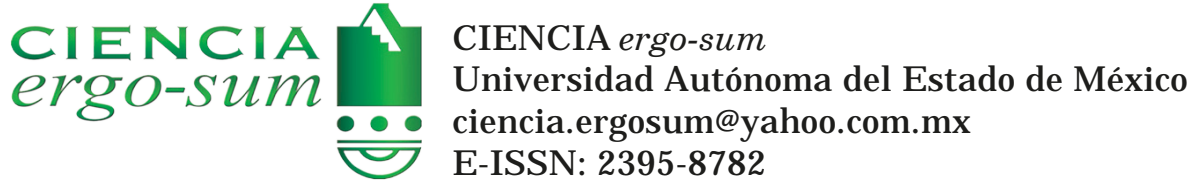

\title{
Gravedad emergente: ¿La llave termodinámica del espacio-tiempo?
}

Sánchez Hernández, Luis Miguel

Gravedad emergente: ¿La llave termodinámica del espacio-tiempo?

CIENCIA ergo-sum, Número especial "Retos de la física no lineal" 2020|e106

Universidad Autónoma del Estado de México, México

Esta obra está bajo una Licencia Creative Commons Atribución-NoComercial-SinDerivar 4.0 Internacional .

Sánchez Hernández, L. M. (2020). Gravedad emergente: ¿La llavetermodinámica del espacio-tiempo?. CIE NCIA ergo-sum, Número especial "Retos de la física no lineal". https://doi.org/10.30878/ces.v27n4a5 


\section{Gravedad emergente: ¿La llave termodinámica del espacio-tiempo?}

Emergent gravity: The thermodynamic key of space-time?

Luis Miguel Sánchez Hernández

Universidad Nacional Autónoma de México, México

Recepción: 21 de agosto de 2019

luis.sanchez@correo.nucleares.unam.mx

(D) https://orcid.org/0000-0003-3867-5583

Aprobación: 10 de febrero de 2020

\section{RESUMEN}

Se exponen algunos aspectos de la incitante relación entre gravedad y termodinámica y cómo ésta ha llevado a proponer que la gravedad es un fenómeno emergente de origen termodinámico-estadístico más que una interacción fundamental. Para desarrollar esta idea, se presenta una breve revisión de los aspectos principales de la termodinámica de agujeros negros y el efecto Unruh. Después, se discute el trabajo de T. Jacobson y se esboza la noción de gravedad como fuerza entrópica propuesta por E. P. Verlinde. Finalmente, se discuten algunas implicaciones que resultan al considerar la gravedad como fenómeno emergente, en particular cómo este nuevo concepto puede ser importante para resolver algunos problemas que los modelos actuales de gravedad no han logrado solucionar.

PAlabras Clave: gravedad emergente, termodinámica de agujeros negros, gravedad cuántica.

\section{Abstract}

We consider some aspects of the inciting relation between gravity and thermodynamics and describe how this has led to propose that gravity is not a fundamental interaction, but an emergent phenomenon with a statistical thermodynamic origin. To develop this idea, first, we briefly review the main aspects of black hole thermodynamics and the Unruh effect. Then, we present the work of T. Jacobson and outline the idea proposed by E. P. Verlinde that gravity is an entropic force. Finally, we discuss some of the consequences that follow from considering gravity as an emergent phenomenon and how this new concept may be important to solve some problems that current gravity models have failed to solve.

KEYWORDS: Emergent gravity, black hole thermodynamics, quantum gravity.

\section{INTRODUCCIÓN}

Hasta ahora los intentos de cuantizar la gravedad han sido guiados por la que es quizá una premisa errónea: asumir que la naturaleza intrínseca de la gravedad es idéntica a la naturaleza de las interacciones electromagnéticas y nucleares. Este posible error puede ser la razón por la que cuantizar la gravedad sea una tarea extremadamente difícil.

La gravedad tiene ciertas características que parecen diferenciarla del resto de interacciones; sin duda, la más notable es su carácter universal, pues cualquier objeto físico está sujeto a su influencia dado que no existe forma de aislarse de un campo gravitacional. Adicionalmente, el principio de equivalencia hace de la masa una carga especial, ya que ninguna otra puede deformar el espacio-tiempo; asimismo, la relación masa-energía también insinúa un carácter especial en la carga gravitacional. Lo anterior en conjunto con el hecho de que la gravedad está relacionada de manera intrínseca con la estructura del espacio-tiempo son aspectos que no deben pasar inadvertidos. Estas propiedades presentes en la gravedad y su carga muestran con claridad aspectos que la excluyen de las otras fuerzas y que posiblemente sea un indicio de que a nivel fundamental son de naturaleza diferente.

Una pista sugerente que sustenta lo dicho se presentó a principios de 1970 cuando se encontró que la gravedad y la mecánica cuántica están estrechamente relacionadas con la termodinámica. Esta conexión fue señalada en primera instancia por Bekenstein, quien postuló que el teorema de no disminución del área de un agujero negro debe tomarse como un enunciado equivalente de la segunda ley de la termodinámica, donde el área debe ser definida como una medida de la entropía de dicho sistema (Bekenstein, 1972; Bekenstein, 1973; Wald, 1999). Más tarde, esta provocativa 
especulación fue demostrada por Hawking al descubrir que, si se consideran efectos cuánticos, un agujero negro puede radiar como si se tratara de un cuerpo caliente (Hawking, 1975). Al poder definirse una temperatura y una entropía para los agujeros negros, es natural suponer que existen grados de libertad microscópicos (Callen, 1985), lo que a su vez lleva a concebir que el propio espacio-tiempo tiene una estructura microscópica! Esta reflexión puede ser clave para entender lo que una teoría cuántica de la gravedad significa. Es decir, quizá el método más adecuado para obtener una teoría microscópica de la gravedad no es aquel en el que se intenta cuantizar un campo, sino más bien uno análogo al procedimiento que lleva a conectar la termodinámica con modelos microscópicos a través de la física estadística. En este sentido, la conexión señalada puede indicar que la gravedad es una teoría efectiva del mismo modo que lo es la termodinámica o la hidrodinámica y, por tanto, tratar de obtener un modelo microscópico mediante un procedimiento de cuantización simplemente no es apropiado. Así, estudiar nuevas posibilidades teóricas es interesante, pues en principio podrían proveer un formalismo para obtener un modelo microscópico de la gravedad que no implica necesariamente un método de cuantización que hasta el momento no ha dado resultados.

Las ideas expuestas en el párrafo anterior han sido objeto de especulación y debate, en especial lo referente a la naturaleza de la gravedad y sus hipotéticos grados de libertad microscópicos (Smolin, 2004; Padmanabhan, 2016). Sin embargo, más allá de esto, lo que es importante es que la gravedad podría ser una teoría independiente del modelo que defina estos grados de libertad microscópicos, lo cual revelaría una característica muy especial que es intrínseca de los sistemas termodinámicos. De ser así, las propiedades gravitacionales podrían ser un resultado efectivo de algún límite continuo, o bien de un promedio estadístico de estos componentes microscópicos. Estas y otras ideas han estimulado la creación nuevos modelos para explicar los fenómenos gravitacionales y han dado pie a postular la posibilidad de que la gravedad sea una teoría termodinámica del espacio-tiempo (Jacobson, 1995; Padmanabhan, 2004; Padmanabhan, 2005; Verlinde, 2010), es decir, que la interacción gravitatoria y el espacio-tiempo en sí mismo son propiedades emergentes.

Es interesante notar que posiblemente estemos delante de un escenario parecido al que se enfrentaron los físicos del siglo XIX cuando al construir una teoría que ordenara y explicara los fenómenos térmicos encontraron relaciones generales entre propiedades macroscópicas de los sistemas que no dependían de la existencia de una estructura microscópica de la materia (Lopez-Monsalvo et al., 2015). Así, por ejemplo, podría ser posible que las ecuaciones de Einstein jueguen el papel de una de estas expresiones generales que relacionan propiedades macroscópicas de sistemas gravitacionales (Jacobson, 1995). De ser el caso, la naturaleza de la gravedad más allá de ser fundamental, en el sentido de las interacciones fundamentales, sería de origen termodinámico-estadístico. Esto podría eventualmente explicar el carácter especial de la gravedad y en definitiva podría ser de muy alta estima para desarrollar una teoría cuántica de la gravedad o incluso podría dar indicios de que tal teoría no existe.

Este trabajo se organiza de la siguiente manera: en la sección 1 se discuten las principales propiedades termodinámicas que presentan los agujeros negros y la importancia que esto ha tenido en la concepción termodinámica de la gravedad. Se consideran principalmente la analogía entre las leyes de la termodinámica y las leyes mecánicas de agujeros negros, además se revisa el efecto Unruh. En la sección 2 se presenta un análisis sobre el trabajo de T. Jacobson, quien, basado en argumentos termodinámicos, logró deducir las ecuaciones de Einstein. Asimismo, se comentan de manera breve los trabajos de T. Padmanabhan y se esbozan las ideas de E. P. Verlinde, quien propone que la gravedad es una fuerza entrópica. Igualmente, se exponen algunas de las críticas que ha se han hecho a su formulación. En la siguiente sección se discuten de manera general las diversas cuestiones que se exponen en este manuscrito. Para finalizar, en la última sección se da una prospectiva acerca de los aportes que se esperan de una formulación de la gravedad como fenómeno emergente.

\section{Termodinámica de aguJERos negros}

Un agujero negro es una configuración espacio-temporal cuya densidad de masa es tan elevada que la curvatura que provoca en el espacio-tiempo es tal que todo objeto que se encuentre a cierta distancia de él es atraído inevitablemente hasta su centro y una vez que algo cae en él jamás podrá salir. Esta clase de objetos astronómicos resultan ser una solución 
de las ecuaciones de campo de la teoría de la relatividad general, y desde su descubrimiento en 1916 ha sido de mucho interés para los físicos teóricos. Su estudio llegó a cierto grado de madurez cuando se estableció el, asíllamado, teorema de no pelo, el cual sostiene que un agujero negro puede ser descrito mediante tres parámetros: su masa $M$, su carga eléctrica $Q$ y su momento angular $J$ (Wald, 1984). Nótese que este hecho es semejante a lo que sucede en la termodinámica, en la cual, para describir un sistema macroscópicamente, se requiere un número muy reducido de variables.

En los años siguientes se encontraron otras características de estos sistemas que fueron realmente inesperados y sorprendentes. Primero, Bekenstein argumenta que el área del horizonte de un agujero negro $A$ debe considerarse como una definición de su entropía (Bekenstein, 1972; Bekenstein, 1973); luego, se demuestra que esta analogía con la segunda ley puede extenderse al resto de leyes termodinámicas al relacionarse con ciertas propiedades mecánicas de los agujeros negros tales como su gravedad superficial $\kappa$ y su momento angular $J$ (Carter et al., 1973). La tabla 1 muestra la analogía completa.

Tabla 1

Analogía entre las leyes de la termodinámica y las leyes mecánicas de agujeros negros

\begin{tabular}{|lcc|}
\hline Ley & Termodinámica & Agujeros negros \\
\hline Cero & $T$ es constante sobre un sistema & $\kappa$ es constante sobre horizontes de agujeros negros estacionarios \\
en equilibrio & $d E=T d S+d W$ & $d M=\frac{\kappa}{8 \pi} d A+\Omega d J$ \\
Segunda & $d S \geq 0$ & $d A \geq 0$ \\
Tercera & No se puede alcanzar $T=0$ & No se puede alcanzar $\kappa=0$ \\
\hline
\end{tabular}

Fuente: Wald, 1984.

De este modo, las cuatro leyes de la mecánica de agujeros negros estacionarios encuentran una profunda correspondencia con las leyes de la termodinámica si consideramos que la gravedad superficial $\kappa$ juega el papel de temperatura, el área del horizonte $A$ el de entropía y la masa $M$ el de energía interna. En el caso de un agujero negro que rota los términos de trabajo están dados por la velocidad angular $\Omega$ y el momento angular $J$, mientras que para un agujero negro con carga, el trabajo está dado en función de su carga $Q$ y el potencial electrostático $\Phi$.

Sin embargo, estas relaciones fueron vistas como meros accidentes formales y la aceptación de los agujeros negros como auténticos sistemas termodinámicos no se dio hasta que Hawking, considerando la teoría de la relatividad general y la teórica cuántica de campos, demuestra que la temperatura y la entropía son cualidades intrínsecas de los horizontes que tienen un significado físico concreto asociado a la posibilidad de que un agujero negro emita radiación (Hawking, 1975; Hawking, 1976). En concreto, Hawking encontró que es posible definir una entropía para un agujero negro dado por la expresión exacta:

$$
S_{B H}=\frac{k_{B} c^{3}}{4 G \hbar} A
$$

donde $G$ es la constante de gravitación, $\hbar$ es la constante de Planck dividida por $2 \pi, c$ es la velocidad de la luz y $k_{B}$ es la contante de Boltzmann.

También encontró que la temperatura de equilibrio para un agujero negro está dada en términos de una cantidad conocida como gravedad superficial $\kappa$ de la siguiente manera:

$$
T_{H}=\frac{\hbar \kappa}{2 \pi k_{B}}
$$

Así, la correspondencia que existía entre las leyes mecánicas de agujeros negros estacionarios y las leyes de la termodinámica pasó de ser una mera coincidencia formal a ser aceptada como una identidad con valor físico. 
No obstante, al aceptar el carácter termodinámico de los agujeros negros necesariamente se sigue que existen grados de libertad microscópicos y, por tanto, se podría inferir que el espacio-tiempo tiene una estructura microscópica que a nivel macroscópico se ve reflejada en expresiones generales que en este caso vienen dadas por leyes dinámicas.

Esto último ha sido una de las ideas conceptuales más importantes de la física teórica contemporánea y ha llevado a sospechar que la gravedad es fundamentalmente de naturaleza termodinámica.

\section{1. Termodinámica de horizontes y efecto Unruh}

Las propiedades termodinámicas en gravitación no son exclusivas de los agujeros negros. Estas propiedades pueden definirse en configuraciones espacio-temporales que presenten horizontes, es decir, para este tipo de sistemas es posible definir una temperatura y una entropía. Fue debido a las investigaciones de W. G. Unruh que se pudo apreciar con claridad esta característica.

W. G. Unruh estudio la teoría cuántica de campos en un espacio-tiempo de Rindler (espacio-tiempo curvo que tiene un horizonte aparente) y descubrió que se puede asociar una temperatura con la aceleración de un observador no inercial (Unruh, 1976; Alsing y Milonni, 2004). Este hecho es conocido como efecto Unruh; en resumen, señala que un observador acelerado notará un baño térmico a una temperatura definida en función de su aceleración, mientras que un observador inercial en las mismas condiciones no percibe nada.

La temperatura que medirá el observador acelerado es idéntica a la que mediría si estuviera inmerso en un baño térmico causado por un cuerpo negro; dicha temperatura está dada por:

$$
T_{U}=\frac{\hbar a}{2 \pi c k_{B}}
$$

donde $a$ es la aceración del observador no inercial.

De esta expresión es claro que si no hay aceleración, la temperatura será nula y, por tanto, un observador que se mueve con velocidad constante o que se encuentra en reposo no mide temperatura alguna.

Por otro lado, el principio de equivalencia afirma que un observador acelerado en un espacio-tiempo plano es equivalente a un observador no acelerado en un campo gravitacional. Así, podríamos apelar a dicho principio y asumir que ahora el observador no se encuentra acelerado sino que está inmerso en un campo gravitacional; de esta manera, la causa de la temperatura de Unruh sería un campo gravitacional. Esto muestra de nuevo una relación muy fuerte entre la termodinámica y la gravedad. Nótese que con esta consideración $(a \sim \kappa)$ la temperatura de Unruh es equivalente a la temperatura de Hawking. Además, recordando que la temperatura de Hawking es la temperatura del horizonte causal de un agujero negro y que tiene un significado físico bien establecido, la temperatura de Unruh posiblemente esté relacionada a su vez con el hecho de que el observador acelerado perciba un horizonte. De esto podemos concluir que los aspectos termodinámicos de la gravedad están relacionados de modo estrecho con los horizontes presentes en algunas configuraciones espacio-temporales.

En el siguiente capítulo se podrá notar que la temperatura de Unruh parece estar muy relacionada con la naturaleza termodinámica de la gravedad y prueba de esto es que encaja de manera perfecta en diversos esquemas de la gravedad como fenómeno emergente.

\section{Gravedad EMERgente}

La idea de la gravedad como fenómeno termodinámico cobró gran relevancia en el panorama de la física teórica con las investigaciones de Hawking y Bekenstein sobre agujeros negros presentadas en la sección anterior. Por un lado, tenemos que estos sistemas son una solución de la teoría de la relatividad general y, por otro lado, un análisis cuántico revela de forma sorpresiva el carácter termodinámico de estos sistemas que por definición son 
el objeto arquetípico en el cual una teoría cuántica de la gravedad podría ser relevante. Con esto puede notarse que la termodinámica parece jugar un papel crucial en la conexión entre la teoría cuántica y la gravitación. Así, es posible que la termodinámica pueda extender un puente entre las dos alejadas orillas de la física teórica. Más aún, quizá sea la llave que abra la puerta que guarda los secretos de la naturaleza del espacio-tiempo y probablemente de una teoría cuántica de la gravedad. Sin embargo, en contraste, también puede ser que descarte en definitiva esta teoría, pues, como apunta Jacobson, si la gravedad resulta ser una propiedad emergente del espacio-tiempo, entonces no tendría sentido tratar de cuantizarla (Jacobson, 1995).

Esta posibilidad ha suscitado una nueva manera, tanto conceptual como formal, de entender los fenómenos gravitacionales y como tal ha promovido un número considerable de investigaciones en las que se abordan diferentes perspectivas teóricas para explicar los aspectos termodinámicos de la gravedad. Entre los trabajos más sobresalientes se encuentran los de T. Jacobson, T. Padmanabhan y E. P. Verlinde.

\section{1. Ecuación de estado del espacio-tiempo}

T. Jacobson fue el primero en exponer de forma clara y objetiva la relación estrecha que existe entre la gravitación y la termodinámica. Su resultado más importante fue demostrar que las ecuaciones de Einstein pueden ser derivadas partiendo de consideraciones termodinámicas y que pueden interpretarse como ecuaciones de estado en el sentido termodinámico (Jacobson, 1995). La idea general de su trabajo se expone en el siguiente párrafo.

Se definen en cada punto del espacio-tiempo horizontes locales de Rindler (es decir horizontes que son percibidos por observadores acelerados). Se considera la temperatura de Unruh $T_{U}$ y un flujo de energía $d Q$ que atraviesa el horizonte, el cual es percibido por el observador acelerado; con esta información y después de un cálculo no trivial, las ecuaciones de Einstein son obtenidas al exigir que la relación de Clausius $d Q=T d S$ se cumpla en el horizonte tomando la entropía proporcional al área de dicho horizonte. Esta derivación lleva a Jacobson a proponer que las ecuaciones de Einstein son equivalentes a una ecuación de estado del espacio-tiempo (Moustos, 2017). En este esquema, lo anterior significa que al espacio-tiempo se le pueden asociar propiedades termodinámicas de tal suerte que cumplen con las relaciones de la termodinámica. Ahora bien, una conclusión importante a la que se llega a partir de este resultado, es que si las ecuaciones de campo de Einstein son equivalentes a una ecuación de estado, entonces intentar cuantizarla de forma canónica es como tratar de cuantizar la ecuación de onda para una onda sonora, lo cual es conceptualmente incorrecto, pues la presión no es una magnitud física fundamental sino más bien una propiedad emergente o colectiva.

Los resultados de Jacobson han sido de mucha importancia para el desarrollo de la idea de la gravedad como un fenómeno termodinámico. En la misma línea de pensamiento, se ha logrado extender sus resultados para teorías gravitacionales que dependen del escalar de Ricci (Elizalde y Silva, 2008) y para teorías generalizadas de la gravedad (Brustein y Hadad, 2009). Por otro lado, estas mismas ideas han sido usadas por Verlinde para desarrollar una teoría en la que propone la gravedad como una fuerza entrópica.

\section{2. Fuerza entrópica}

En esta sección se introduce la noción de fuerza entrópica, la cual es central en la formulación de Verlinde. Se define una fuerza entrópica como aquel tipo de interacción que resulta de la tendencia de un sistema a evolucionar hacia estados de mayor entropía de acuerdo con la segunda ley de la termodinámica. Es decir, es una interacción que no involucra agentes que causen el movimiento, sino que este es un fenómeno emergente que es consecuencia directa de cambios en la entropía.

Considerando un sistema termodinámico en el que el trabajo hecho por una fuerza $F$ está dado por $d W=F d x$, donde $d x$ es un desplazamiento, y dado el calor en términos de la temperatura $T$ y la entropía $S, d Q=T d S$, de la primera ley de la termodinámica, se sigue que 


$$
T d S=d U-F d x
$$

La ecuación de Gibbs para esta relación implica (Müller, 2007):

$$
F=\frac{\partial U}{\partial x}-T \frac{\partial S}{\partial x}
$$

Esta expresión muestra claramente una contribución energética y una contribución entrópica. En casos donde la contribución energética es despreciable respecto a la contribución entrópica o cuando la energía no depende de la distancia se dice que $F$ es una fuerza entrópica.

Es importante aclarar que fuerzas de este tipo no debe entenderse en el sentido Newtoniano en el que se asume que un agente es el que produce un cambio. En el caso de una fuerza entrópica no hay agente externo que produzca cambio, sino que éste se presenta simplemente como una consecuencia de la evolución del sistema que por naturaleza tiende a ir a estados con entropía más alta. Fuerzas de este tipo se presentan, por ejemplo, en la biología.

\subsection{Gravedad como fuerza entrópica}

Considerando que la gravedad es un fenómeno emergente en lugar de una interacción fundamental, E. P. Verlinde introduce la noción de gravedad como fuerza entrópica (Verlinde, 2010). Mediante esta concepción, y asumiendo algunos supuestos generales como el principio de equipartición de la energía, el principio holográfico y el efecto Unruh, deriva la segunda ley de Newton, la ley de gravitación Universal y las ecuaciones de Campo de Einstein.

El concepto más importante y novedoso que presenta es la idea de tomar la gravedad como un efecto colectivo de origen termodinámico-estadístico que resulta de la tendencia de un sistema hacia configuraciones particulares que maximicen su entropía. De esta manera, lo que percibimos como fuerza entre masas no es más que una redistribución de la materia de acuerdo con la segunda ley de la termodinámica.

La secuencia lógica que lleva a Verlinde a derivar la segunda ley de Newton se basa en tres consideraciones:

I) Una fuerza entrópica está dada por una expresión del tipo:

$$
F=T \frac{\Delta S}{\Delta x}
$$

II) La contribución a la entropía de un horizonte aumenta por una cantidad $\Delta S=2 \pi k_{B}$ cuando una partícula se encuentra a una distancia de una longitud de Compton $\Delta x=\hbar / m c$.

III) Se supone que el efecto Unruh puede ser tomado en sentido inverso, es decir, $T$ es la temperatura necesaria para producir una aceleración $a$.

Al sustituir las magnitudes para $\Delta S, \Delta x$ y $T$ en la expresión para la fuerza entrópica, se obtiene:

$$
F=\left(\frac{\hbar a}{2 \pi c k_{B}}\right)\left(\frac{2 \pi m c k_{B}}{\hbar}\right)=m a
$$

Este resultado sugiere que el movimiento de una masa es originado por un gradiente de entropía que está definido entre las pantallas holográficas, las cuales pueden ser entendidas como una especie de horizontes locales, que, de manera análoga a la idea de Jacobson, están definidas en cada punto del espacio-tiempo y a las cuales se les puede asociar una entropía y una temperatura. Así, en última instancia, la segunda ley de Newton puede ser interpretada como una fuerza termodinámica!

De igual forma, la ley de gravitación de Newton puede derivarse bajo este esquema. Para esto, Verlinde apela a una versión del principio holográfico (Susskind, 1995) que básicamente consiste en asumir que la información referente al movimiento de los cuerpos está almacenada en las pantallas holográficas, y la cual es proporcional al 
área $A$ de dichas pantallas (de la misma manera que la entropía de un agujero negro es proporcional al área del horizonte); asimismo, postula que los $N$ bits codificados en un horizonte obedecen la relación (Granger, 2010):

$$
N=\frac{A c^{3}}{G \hbar}
$$

Adicionalmente, supone que el principio de equipartición de la energía $E=1 / 2 k_{B} N T$ se satisface en las pantallas holográficas (horizontes locales) y que por tanto es posible definir la temperatura como:

$$
T=\frac{2 G \hbar E}{A c^{3} k_{B}}
$$

Y de nuevo usando la expresión (6) para la fuerza entrópica se llega a la expresión:

$$
\begin{aligned}
F & =\left(\frac{2 G \hbar E}{A c^{3} k_{B}}\right)\left(\frac{2 \pi m c k_{B}}{\hbar}\right) \\
& =\left(\frac{4 \pi G m E}{A c^{2}}\right)
\end{aligned}
$$

Posteriormente, tomando $A=4 \pi R^{2}$, es decir, que las pantallas son círculos de radio $R$ y la relación masa-energía $E=M c^{2}$, llegamos a la expresión de la ley de gravitación de universal:

$$
F=G \frac{M m}{R^{2}}
$$

Notemos que una fuerza entrópica no genera un aumento de la entropía, sino que por el contrario es la tendencia de la entropía a crecer, según la segunda ley de la termodinámica, lo que genera la fuerza entrópica. De esta manera, la gravedad no induce un cambio en la entropía del sistema (la gravedad es reversible), sino que la tendencia natural del sistema hacia estados de mayor entropía es la que genera una interacción (fuerza entrópica) que redistribuye la materia y es esto justamente lo que percibimos como la fuerza gravitacional.

Esta derivación sugiere que la gravedad debe considerarse como una interacción de naturaleza termodinámica resultante de un gradiente de entropía entre las pantallas holográficas que permean todo el espacio y que por tanto debe calificarse como un fenómeno emergente!

Por otro lado, considerando argumentos relativistas para generalizar su esquema teórico y siguiendo un razonamiento análogo al de Jacobson, Verlinde fue capaz de derivar las ecuaciones de la relatividad general. No obstante, como Verlinde acepta en su artículo, lo importante de esto no es que haya podido derivarlas, porque esto ya lo había hecho Jacobson, sino más bien su aporte está en lograr identificar a los gradientes de entropía como un mecanismo mediante el cual el espacio-tiempo y la gravedad emergen.

\subsection{Objeciones a la formulación de gravedad como fuerza entrópica}

La explicación de la gravedad dada por Verlinde es una idea muy estimulante, aunque, como él mismo acepta, es una construcción heurística e incompleta por el momento. Como tal, ha sido objeto de múltiples críticas que señalan inconsistencias. Una de las críticas más serias fue hecha por A. Kobakhidze, quien en su trabajo 
(Kobakhidze, 2011) expone un punto en el que la teoría de Verlinde es insatisfactoria. Su argumento es que el concepto de gravedad como fenómeno emergente y la visión tradicional sugieren predicciones diferentes sobre la interacción entre gravedad y partículas cuánticas. En 2002, V. V. Nesvizhevsky y sus colaboradores (Nesvizhevsky et al., 2002) realizaron experimentos en el contexto de interferometría de neutrones en el campo gravitacional de la tierra que mostraron cómo es la interacción de estas partículas cuánticas en un campo gravitacional. Considerando las conclusiones de dichos experimentos, Kobakhidze señala que la teoría de Verlinde no concuerda con estos resultados y que por tanto no puede ser correcta en su forma actual.

Los experimentos muestran que los neutrones interfieren consigo mismo creando patrones de interferencia (tal como en el caso de experimentos con doble rendija) y que el efecto de la gravedad puede apreciarse al distinguir que la localización del patrón se modifica de acuerdo con la teoría al asumir la función de onda en un campo gravitacional. Así, el desplazamiento del patrón de interferencia puede explicarse asumiendo que el efecto de la gravedad es cambiar la fase de la función de onda. El punto central de la crítica de Kobakhidze está basado en este resultado y puede resumirse de la siguiente manera: el campo gravitacional interactúa con los neutrones causando un corrimiento en el patrón de interferencia que no destruye dicho patrón, es decir, conserva la coherencia del sistema. En contraste, en el caso de la propuesta de Verlinde, la interacción de los grados de libertad que permiten definir la entropía en las pantallas holográficas y que dan origen a la fuerza entrópica destruirían el patrón de interferencia al interactuar con los neutrones contradiciendo los resultados experimentales. Éste es ciertamente un punto que la teoría de Verlinde en su estado actual no puede responder del todo. Aun cuando no está claro que los grados de libertad deban interactuar con los neutrones, en el caso en que dicha interacción exista, debe ser tal que el patrón de interferencia no sea destruido. En esta dirección se han presentado algunos trabajos tratando de explicar las inconsistencias que presenta la formulación de gravedad como fuerza entrópica (Chaichian et al., 2011).

Otro aspecto importante que ha sido criticado es la irreversibilidad que conlleva la idea de gravedad como fuerza entrópica. En la versión newtoniana la gravedad es una fuerza central y, por ende, reversible. En el modelo de Verlinde, sin embargo, se considera que esta es una fuerza entrópica, es decir, que el movimiento de una masa debido a esta fuerza va siempre en la dirección creciente de entropía y, por lo tanto, se vuelve un proceso irreversible. Esto parece introducir una inconsistencia en la teoría. No obstante, aunque pueda parecer contraintuitivo, es posible tener fuerzas entrópicas que sean conservativas (Visser, 2011). El caso más conocido es el de un polímero inmerso en un baño térmico cuya entropía y su tendencia natural hacia estados que la maximicen, conducen a una relación aproximada de la ley de Hook (Müller, 2007). De esa manera, la irreversibilidad es una objeción válida, pero no parece ser una inconsistencia insalvable y posiblemente pueda ser explicada al hacer consideraciones físicas adecuadas si bien éstas pueden imponer restricciones adicionales a la teoría (Visser, 2011).

No obstante las críticas que ha recibido, su artículo ha despertado un gran interés en el tema de la gravedad como fenómeno emergente al mismo tiempo que ha estimulado la creación de muchos trabajos en esa y otras direcciones. Por otro lado, existen notables que abordan el tema desde perspectivas completamente diferentes. Entre las más importantes se encuentran las múltiples investigaciones de Padmanabhan, de las cuales una de las más destacadas es aquella en la que muestra una relación holográfica entre el término de volumen y el término de superficie en la acción de Einstein-Hilbert (EH) (Padmanabhan, 2005). Padmanabhan argumenta que el término de superficie en la acción EH puede construirse a partir de nociones generales y, partiendo de éste y dada su relación con el término de volumen, es posible derivar las ecuaciones de Einstein a partir de consideraciones termodinámicas. Con esto, Padmanabhan concluye que los grados de libertad microscópicos están definidos en el término de superficie de la acción EH y que la gravedad es intrínsecamente holográfica. Además, ha discutido aspectos conceptuales importantes (Padmanabhan, 2010) y ha formulado analogías entre rasgos de la gravedad y propiedades elásticas de sólidos (Padmanabhan, 2004). Otro trabajo relevante puede ser consultado en Cai y Kim (2005) y Fei-Quan Tu et al. (2018). En particular, el autor de este artículo se encuentra desarrollando una nueva 
perspectiva de abordar la gravedad como fenómeno emergente, la cual se puede resumir de la siguiente manera: es bien conocido que las ecuaciones de Einstein establecen una conexión entre la gravedad y la geometría. De manera análoga, es posible encontrar una conexión entre termodinámica y geometría. La Geometrotermodinámica (GTD) es un formalismo geométrico de la termodinámica (Quevedo 2007) que permite analizar mediante las herramientas de la geometría diferencial las propiedades termodinámicas de un sistema. Así, la idea es que usando GTD se puede asociar un espacio termodinámico (espacio de equilibrio) a cualquier solución de las ecuaciones de Einstein que tenga horizonte, por ejemplo, agujeros negros y modelos cosmológicos. De ahí, nuestra propuesta de gravedad emergente consiste en tomar ese espacio termodinámico y derivar de éste la métrica del espacio-tiempo de forma tal que las coordenadas espacio-temporales resulten ser funciones de las variables termodinámicas, es decir, dichas coordenadas "emergen” de la información termodinámica.

\section{Discusión Y CONCLUSIONES}

La búsqueda de una teoría cuántica de la gravedad es probablemente el reto más importante que la física teórica ha abordado en el último siglo. Sin embargo, los avances son más bien pocos y, en mi opinión, esto se debe a una limitación de los conceptos en los que fundamos nuestro conocimiento más que a una cuestión matemática. Lo que quiero decir con esto es que para resolver los problemas a los que la física teórica se enfrenta hoy, más que un replanteamiento o refinamiento matemático, es necesaria una reformulación conceptual en la forma en que pensamos el mundo. Las teorías de la mecánica cuántica y la relatividad fueron en su momento tomadas con recelo porque exigían una manera completamente diferente de concebir la realidad; no obstante, estas teorías lograron dar cuenta de un gran número de resultados que la física de su tiempo no podía explicar de forma satisfactoria. De no haberse aceptado estas nuevas teorías y sus implicaciones, probablemente la física se hubiera estancado por mucho tiempo. No obstante, a pesar de todas las contribuciones que estas teorías han legado a la ciencia, hoy parece que sus nociones ya no son del todo suficientes para explicar el universo. Es necesario, por tanto, hacerse de nuevos conceptos que nos permitan avanzar ahí donde los conceptos del siglo pasado ya no lo permiten. En esta dirección, los nuevos conceptos e ideas que se han venido formulando a partir del descubrimiento de la termodinámica del agujero negro pueden colocarse como una herramienta conceptual importante que permita dar cuenta de algunos problemas que hasta hoy siguen sin solución.

Al descubrir que un agujero negro posee entropía y temperatura se advirtió la existencia de una profunda relación entre gravedad y termodinámica. Posteriormente, múltiples investigaciones han profundizado en esta relación y han proyectado resultados que la respaldan en mayor medida. Dichas investigaciones han llevado a plantear la noción de que la gravedad puede ser una propiedad emergente del espacio-tiempo y por ende una interacción cuya naturaleza difiere del resto de interacciones fundamentales. T. Jacobson demostró, en un trabajo sobresaliente, que las ecuaciones de Einstein pueden ser derivadas a partir de consideraciones termodinámicas, las cuales pueden ser interpretadas como ecuaciones de estado en el sentido termodinámico. Por otro lado, E. P. Verlinde postuló que la gravedad es una fuerza entrópica, dando lugar así a una de las formas más incitantes de entender la gravedad. En su trabajo muestra que al tomar esta idea de la gravedad, y asumiendo la validez del efecto Unruh, el principio holográfico y la equipartición de energía, es posible derivar las leyes de Newton (segunda y gravitación universal) y, al hacer una generalización relativista, también es factible derivar las ecuaciones de Einstein mostrando con esto que la gravedad acepta una interpretación termodinámica. Por otro lado, Padmanabhan y otros también han obtenido resultados que apoyan la idea de gravedad como fenómeno emergente. Así, estas ideas no deberían considerarse más como una curiosidad, más bien como resultados que sugieren que la interacción gravitacional no es realmente interacción fundamental sino de origen termodinámico-estadístico. Esto implicaría que la noción continua del espacio-tiempo es, a su vez, un efecto a gran escala y que algunas de las variables espacio-temporales son variables colectivas, por lo que intentar una cuantización de estas magnitudes no es apropiado. 
Si bien por el momento las diversas formulaciones de gravedad como fenómeno emergente pueden considerarse incompletas, y en algunos casos inconsistentes, lo cierto es que han mostrado un camino alternativo que parece prometedor.

\section{Prospectiva}

Existen al menos dos puntos en los que se espera que la noción de gravedad como fenómeno emergente pueda contribuir:

a) Una comprensión más profunda de la gravedad: es posible que los problemas que las teorías actuales de la gravedad no pueden responder se deban a una comprensión deficiente de la naturaleza de la gravedad. Prueba de esto es que hay fenómenos que no somos capaces de explicar de forma con las teorías actuales de gravitación. Entre ellos, la materia obscura figura como uno de los principales problemas. El concepto de materia obscura se introdujo, entre otras cosas, para explicar el problema de las llamadas curvas de rotación de las galaxias, el cual básicamente consiste en que las observaciones de las velocidades de rotación de las galaxias no coincide con lo que predice la teoría, lo cual sugiere que la interacción gravitacional que muestran las galaxias es mayor de la que deberían exhibir dada la estimación de la cantidad de materia que contienen. Dichas desviaciones pueden explicarse al suponer la existencia de un cierto tipo de materia que curva el espacio-tiempo pero que no interactúa con la radiación electromagnética.

Se han propuesto numerosos modelos que intentan explicar la materia obscura; sin embargo, hasta ahora todos son insatisfactorios hasta cierto punto. La mayoría de estos modelos se basan en modificaciones de la visión Newtoniana o Einsteiniana de la gravedad, lo cual, a mi parecer, no es del todo adecuado. ¿Por qué introducir un nuevo tipo de materia? ¿Por qué no pensar que este efecto que se observa es una propiedad de la gravedad que aún desconocemos? Para responder a estas preguntas probablemente tengamos que reconocer que seguimos aferrándonos a una visión de la realidad que podría no ser correcta y aun así esperamos que ésta nos proporcione resultados correctos. No obstante, para avanzar necesitamos mirar al futuro con una visión más amplia y considerar nuevas formulaciones teóricas y conceptuales que nos permitan explorar otras alternativas.

En esta dirección, E. P. Verlinde ha demostrado que tomar la gravedad como un fenómeno emergente es una forma viable de solucionar algunos de estos problemas. Con su investigación (Verlinde, 2016) puede dar cuenta de las desviaciones en los movimientos de las galaxias sin verse en la necesidad de introducir materia obscura, sino que este efecto resulta de ciertas propiedades nuevas que surgen al suponer la gravedad y el espacio-tiempo como fenómenos emergentes. Con este resultado se muestra que considerar teorías alternativas de la gravedad puede ser la dirección correcta a seguir para futuras investigaciones. En particular, se espera que si la formulación de la gravedad como un fenómeno emergente es correcta, entonces proporcione una comprensión más profunda de la naturaleza de la gravedad, lo que a su vez naturalmente permitiría encontrar soluciones de algunos problemas de la física contemporánea.

b) Problema de gravedad cuántica: reconciliar la mecánica cuántica con la gravedad es uno de los retos más grandes que la física teórica enfrenta.

Desde el siglo pasado la búsqueda de una teoría microscópica de la gravedad ha ocupado un lugar fundamental en la actividad de los físicos teóricos; sin embargo, hasta ahora no da dado resultados contundentes. Aun así, existen resultados que sugieren un camino que podría reconciliar la teoría cuántica con la gravitación. Las investigaciones de Hawking sugirieron una relación entre gravedad, termodinámica y la teoría cuántica. La ecuación (1) manifiesta de manera explícita esta relación al tener en una misma expresión las constantes fundamentales que representan dichas teorías. Dado que esta coexistencia aparentemente puede ser sostenida al considerar aspectos termodinámicos, se espera que la termodinámica pueda jugar un papel 
crucial en la construcción de una formulación microscópica de la gravedad que la reconcilie con la teoría cuántica. En particular, considerar que la gravedad tiene un origen termodinámico-estadístico podría ser la llave para entender lo que una teoría microscópica de la gravedad significa. Asimismo, es preciso señalar que si bien este nuevo concepto de gravedad puede dar valiosas pistas que permitan construir una teoría cuántica de la gravedad, también es posible que descarte la existencia de dicha teoría. De ser este el caso, como sugieren las investigaciones que aquí presentamos, podríamos estar ante una teoría alternativa de la gravitación que revolucione nuestra concepción del universo y que posiblemente pueda dar solución a las preguntas que por años se ha esperado que una teoría cuántica de la gravedad responda.

\section{Agradecimientos}

Deseo agradecer al doctor Hernando Quevedo por sus valiosas observaciones y por su apoyo en la elaboración de este manuscrito, así como también al doctor Máximo Agüero por su amable invitación a colaborar en esta revista.

\section{REFERENCIAS}

Alsing, P. M., \& Milonni, P. W. (2004). Simplified derivation of the Hawking-Unruh temperature for an accelerated observer in vacuum. American Journal of Physics, 72, 1524-1529. arXiv:quantph/0401170v2.

Bekenstein, J. D. (1972). Black holes and the second law. Letters Nuovo Cimento, 4, 737.

Bekenstein, J.D. (1973). Black holes and entropy. Physical Review D, 7, 2333.

Brustein, R., \& Hadad, M. (2009). Einstein equations for generalized theories of gravity and the thermodynamic relation $d Q=T d S$ are equivalent. Physical Review Letters, 103, 101301.

Cai, R. G., \& Kim, S. P. (2005). First law of thermodynamics and Friedmann equations of Friedmann-Robertson-Walker Universe. Journal of High Energy Physics, 2, [hep-th/0501055].

Callen, H. B. (1985). Thermodynamics and an introduction to thermostatics. John Wiley and Sons Inc.

Carter, B., Hawking, S. W., \& Bardeen, J. M. (1973). The four laws of black hole mechanics. Communications in Mathematical Physics, 31, 161-170.

Chaichian, M., Oksanen, M., \& Tureanu, A. (2011). On gravity as an entropic force. Physics Letters B, 702, 419-42. arXiv:1104.4650 [hep-th].

Elizalde, E. y Silva, P. J. (2008). f(R) gravity equation of state. Physical Review D, 78, 061501.

Fei-Quan Tu, Yi-Xin Chen, Bin Sun, \& You-Chang Yang. (2018). Accelerated expansion of the universe based on emergence of space and thermodynamics of the horizon. Physics Letters B, 784, 411. arXiv:1707.06461 [gr-qc].

Granger, A. (2010). Thermodynamic gravity and the emergence of space with geometry (QFFF Dissertation). London: Imperial College. https://www.imperial.ac.uk/media/imperial-college/research-centresand-groups/theoretical-physics/msc/dissertations/2010/Andrew-Granger-Dissertation.pdf

Hawking, S. W. (1976). Black holes and thermodynamics. Physical Review D, 13, 191-197.

Hawking, S. W. (1975). Particle creation by black holes. Communications in Mathematical Physics, 43, 199-220.

Jacobson, T. (1995). Thermodynamics of space-time: The Einstein equation of state. Physical Review Letters, $75,1260-1263$. 
Kobakhidze, A. (2011). Gravity is not an entropic force. Physical Review D, 83, 021502. arXiv:1009.5414 [hep-th]

Lopez-Monsalvo, C. S., Quevedo, H., \& Bravetti, A. (2015). Maximally symmetric spacetimes emerging from thermodynamic fluctuations. arXiv: $1503.08358 \mathrm{v} 2$ [gr-qc].

Moustos, D. (2017). Gravity as a thermodynamic phenomenon (M. Sc. Thesis). Greece: University of Patras. arXiv:1701.08967v1 [gr-qc].

Müller, I. (2007). A history of thermodynamics: The doctrine of energy and entropy. New York: Springer.

Nesvizhevsky, V. V., Boerner, H. G., \& Gagarsky, A. M. (2002). Quantum states of neutrons in the Earth's gravitational field. Nature, 415, 297-299

Padmanabhan, T. (2004). Gravity as elasticity of spacetime: A paradigm to understand horizon thermodynamics and cosmological constant. International Journal of Modern Physics, 13, 2293-2298. arXiv:gr-qc/0408051.

Padmanabhan, T. (2005). Holographic gravity and the surface term in the Einstein-Hilbert action. Brazilian Journal of Physics, 35, 362. arXiv:gr-qc/041206810]

Padmanabhan, T. (2010). Thermodynamical aspects of gravity: New insights. Reports on Progress in Physics, 73, 046901. arXiv:0911.5004v2 [gr-qc].

Padmanabhan, T. (2016). Journal of Physics: Conference Series, 701, 012018

Quevedo, H. (2007). Geometrothermodynamics. Journal of Mathematical Physics, 48, 013506.

Sánchez, A., Vázquez, A., \& Quevedo, H. (2015). Relativistic like structure of classical thermodynamics. General Relativity and Gravitation, 47(4), 36.

Smolin, L. (2004). Atoms of space and time. Scientific American, 290(1), 66-75.

Susskind, L. (1995). The world as a hologram. Journal of Mathematical Physics, 36, 6377. arXiv:hep-th/9409089.

Unruh, W.G. (1976). Notes on black hole evaporation. Physical Review D, 14, 870.

Verlinde, E. P. (2010). On the origin of gravity and the laws of newton. arXiv:1001.0785.

Verlinde, E. P. (2016). Emergent gravity and the dark universe. arXiv:1611.02269

Visser, M. J. (2011). Conservative entropic forces. Journal of High Energy Physics, 140.

Wald, R. M. (1984). General relativity. Chicago: The University of Chicago Press.

Wald, R. M. (1999). Gravitation, thermodynamics, and quantum theory. Classical and Quantum Gravity,16, A177-A190. arXiv:gr-qc/9901033.

CC BY-NC-ND 